\title{
El nuevo entorno educativo de Bolonia: ágoras transformadas por el esquema Tecno-Holista
}

\author{
Irene Melgarejo Moreno \\ Universidad Católica San Antonio de Murcia \\ imelgarejo@ucam.edu \\ María del Mar Rodríguez Rosell \\ Universidad Católica San Antonio de Murcia \\ mmrodriguez@ucam.edu
}

\begin{abstract}
Resumen:
Introducir nuevos procesos de comunicación en el aula transforma sustancialmente el papel del docente, que deja de ser un altavoz educativo para convertirse en un guía dentro del proceso de enseñanza/ aprendizaje. Este nuevo reto provoca dos consecuencias: la necesidad de una metodología para afrontar las nuevas culturas de la comunicación y la transformación en diseño y configuración de la nueva ágora universitaria. En este artículo se incluye la propuesta de un ejemplo práctico de ágora adaptada a las nuevas demandas de la Sociedad Multipantallas donde se muestran las posibilidades del buen uso de los medios de comunicación aplicados a la docencia.
\end{abstract}

Palabras clave: comunicación; docencia; medios; aula y educación.

\section{New education of Bologna: transformed classroom by Techno-holistic paradigm}

\begin{abstract}
:
New communication processes transform teacher's role that becomes a guide in the process of teaching/ learning. This new challenge causes two consequences: the need for a methodology to meet new cultures of communication and transformation in design of the new university classroom. This article includes a proposal for a practical example of agora adapted to the new demands for society which shows the possibilities for good use of the media applied to teaching.
\end{abstract}

Key Words: communication; teaching; media; classroom and education.

\section{Referencia normalizada:}

Melgarejo Moreno, I. y Rodríguez Rosell, M.M. (2014): El nuevo entorno educativo de Bolonia: ágoras transformadas por el esquema Tecno-Holista. Historia y Comunicación Social. Vol. 19. Núm. Especial Enero. Págs. 797-812.

Sumario: 1. La visión tecno-holista como evolución del clasicismo educativo. 1.1. Revisión, evolución y transformación de los esquemas educativos clásicos 1.2. El paradigma tecno-holista como una forma de completar un estilo educativo para la vida. 2. Principales características educativas del Espacio Europeo de Educación Superior. 2.1. Las novedades introducidas por el Espacio Europeo de Educación Superior. 2.2. La creación del Espacio Virtual Europeo de Enseñanza Superior. 2.3. Las herramientas docentes en la Ágora Digital universitaria. 3. A modo de conclusión. 4. Bibliografía 


\section{La visión tecno-holista como evolución del clasicismo educativo}

En la actualidad se apuesta por un sistema educativo propio y particular; un sistema al que indiscutiblemente se ha llegado tras superar las diferentes y variadas teorías de la educación o corrientes pedagógicas que han venido ocupando épocas anteriores. La verdad es que esos paradigmas educativos a los que nos referimos "no han tenido un desarrollo sencillo" (Flórez, 1999), sobre todo si pensamos en las constantes tensiones que se encuentran entre las instituciones educativas (seguramente más conservadoras) y otras corrientes más innovadoras que apuestan por un entorno educativo más novedoso. Es cierto que el objetivo de este epígrafe es defender las bondades de un nuevo modelo de paradigma educativo acorde a la actualidad y su realidad cambiante, pero no tendría sentido olvidar la evolución y transformación de modelos anteriores que, basados en las principales corrientes pedagógicas, han ayudado a dar valor a los más novedosos avances en el entorno de la enseñanza y el aprendizaje. Mirar hacia atrás y "beber" de la Historia es siempre un ejercicio recomendable porque ayuda a reconocer con claridad los perfiles de los objetos de estudio, sus limitaciones y sus posibilidades, sus puntos fuertes y sus puntos débiles, etc. En definitiva, una visión al pasado nos ayuda a entender la estructura de un nuevo modelo que estamos convencidos se debe instalar en las experiencias educativas actuales, y es por eso que a continuación se hará un breve pero necesario repaso de los principales paradigmas educativos que han conformado el panorama en épocas anteriores.

\subsection{Revisión, evolución y transformación de los esquemas educativos clásicos}

Arrancamos en la antigua Grecia donde la democracia participativa era la apuesta clara y donde argumentar las diferentes temáticas de forma contrastada y contrapuesta era lo más habitual. Este simple hecho supone, tal y como se proponía en el agón de las obras de teatro clásico griego, un desafío, un enfrentamiento provocado. Recordemos que en el agón se anunciaba un debate formal entre dos personajes y el coro, que en algunas ocasiones se ha representado por la gente en la propia obra, que actuaría de juez. La importancia de este apunte radica en el hecho de focalizar los esfuerzos en el concepto de educación completa (no parcial), equilibrando los saberes entre las principales disciplinas (aritmética, música y deportes) y no perdiendo de vista uno de los fines fundamentales de su modelo que recaía en el deseo de educar para convertirse en buenos ciudadanos. La visión cósmica del modelo empaña otras cuestiones y permite que el maestro, bien los paidagogos o los mentores, se personalicen en el reflejo de la cultura, del saber, del saber hacer y del saber ser, para conducir a sus discípulos a la excelencia y alcanzar la areté.

La configuración de este modelo atiende a las características evolutivas tanto del Viejo como del Nuevo Paradigma, de modo que nos basamos para aprender tanto en el individualismo, en lo cuantitativo y en la atomización del sistema tres características propias del viejo modelo, como de la sociedad, lo cualitativo y el carácter holístico de la educación del modelo más nuevo, sin olvidar características propias de los modelos jerarquizados propios del viejo paradigma a los modelos flexibles de los 
más novedosos, de los sistemas piramidales a las organizaciones espontáneas entre iguales, entre otras.

Continuando con la visión de los paradigmas educativos tradicionales recordaríamos, la que a nuestro entender es una de las ternas más utilizadas y que incluye a los paradigmas positivista, interpretativo y crítico. En el fondo, estos tres no dejan de ser sino el resultado de la interpretación que sobre el sistema educativo ofreció Habermas en su obra Conocimiento e interés (1968), que dijo -tal y como recogen los autores Ayuste y Trillo (2005) - que:

El conocimiento tiene que ver con las necesidades o intereses que mueven a las personas a conocer, $y$ tales intereses pueden ser de tres tipos: el interés técnico motivado por el deseo de dominar la naturaleza, el interés práctico que es el que lleva a las personas a entenderse $y$, por último, el interés emancipatorio propio de las teorías críticas que pretenden conocer la realidad para transformarla (222).

Es verdad que son paradigmas que sitúan su origen, tal vez lejos del entorno pedagógico, aunque no se puede negar ni una conceptualización clara ni una calidad intelectual evidente en ellos. Son paradigmas sencillos de entender, con facilidad de implementación (sobre todo a lo que a teorías abstractas se refiere) pero difíciles de nominar ${ }^{1}$. Y son paradigmas que se han mantenido firmes en el devenir educativo, sufriendo, eso sí, variación en su terminología o ligeros cambios conceptuales. Por eso podemos hablar del paradigma tecnológico (o conductista, como propone Colom (2002) en La (de) construcción del conocimiento pedagógico) para referirnos al positivista, del paradigma hermeneútico para referirnos al interpretativo o al paradigma dialéctico para referirnos al crítico. Son muchos los autores, entre los que nos posicionamos, que preferimos considerar al componente tecnológico como uno de los presentes - en mayor o menor medida- en todos ellos.

Podríamos seguir haciendo un recorrido por las propuestas pedagógicas que se han establecido en los diferentes periodos de la historia reciente, y así podríamos citar a la Escuela Nueva. Esta tendencia pedagógica capitaneada por John Dewey (18591952) propuso que la educación debería tener como eje principal a los intereses de los alumnos e insistió en acercar el aprendizaje a través de la práctica más que a través de componentes teóricos. De igual forma, la Pedagogía Liberadora, que se sitúa de forma clara en el entorno revolucionario de ideas surgidas en la década de los setenta del pasado siglo en países de América Latina, basa y centraliza su propuesta en el entorno del alumnado.

Otra de las corrientes pedagógicas destacadas es la denominada como Enfoque Cognitivo, que tiene como máximo exponente al Constructivismo. El primero, con Jean Piaget (1896-1980) explicaría la evolución educativa de los más pequeños a través de funciones básicas como la acomodación y la asimilación. Pero lo verdaderamente relevante es el hecho de que las implicaciones educativas de esta corriente se detectan en las aulas en momentos muy variados como los creativos, reflexivos o de resolución de problemas (de gran valor si pensamos en los modelos paradigmáticos más actuales). 
Resulta interesante el modelo propuesto por la teoría Sociohistórica, que apunta algunos de los criterios que actualmente son tenidos en cuenta en el paradigma actual. Propio del paradigma cualitativo, las actividades sociales en las que los individuos participan, configuran y moldean la ideología de la sociedad en cuestión. Según Vigotsky todo proceso debe ser estudiado desde el punto de vista de la interacción con el medio, teniendo en cuenta el contexto histórico y cultural.

Una de las grandes diferencias paradigmáticas tal vez recaiga en la disparidad entre los modelos cuantitativos y cualitativos. En el paradigma actual, más ligado a la técnica que ningún otro y adecuado a una filosofía holista, como se verá más adelante, encontramos principios de ambos: desde el conocimiento fundamentado en los hechos en el que se apoya la Ciencia (que demuestra su objetividad) hasta la subjetividad enriquecedora de la contextualización de esos mismos hechos, que va a permitir construir teorías a partir de la práctica. Podríamos añadir al respecto que ya otros teóricos relevantes como Dewey invitaban a reflexionar sobre la eficacia de la "lectura distante" de los objetos de estudio. El mismo Mcluhan (Ulloque, 2009: 4) decía: "no sé quién descubrió el agua por primera vez, pero estoy seguro que no fueron los peces" en un proceso se vuelven incapaces de describirlo". También Ulloque (2009) refuerza esta idea insistiendo en que "los estudiosos de los hechos humanos necesitan separarse de los fenómenos que desean explicar".

Como se puede comprobar, todos los modelos propuestos aportan alguna novedad respecto a los anteriores, pero no dejan de presentar elementos recurrentes. No olvidemos, no obstante, que si pensamos en aprender, la tendencia es darle importancia al cómo más que al qué, que aunque sigue manteniendo su estatus, aparece diluido por el propio proceso. Dice la antropóloga Montagu (1958) “en la enseñanza, el método, y no el contenido, constituye el mensaje", y añade que "el proceso de culturizar a una persona no depende de la transmisión del conocimiento, sino de la manera en la que el profesor lo transmite" (62). Pero por otro lado, $-\mathrm{y}$ esto sería motivo de reflexión profunda- tal y como afirma Manuel Benito (2009) "el problema esencial no es tanto si usar o no las herramientas, como la perspectiva y la finalidad con las que se usan".

Por eso somos capaces de entender el paradigma de la Modernidad como un proceso vivo en el que interviene el individuo a través de la autorreflexión con la que se es capaz de transformar no solo el conocimiento sino a la sociedad; es un concepto humanista, urbano, individualista... que se acerca al holismo que enarbola el paradigma más actual.

Terminaremos hablando del modelo Postmodernista que redirecciona la educación hacia el mundo de las nuevas tecnologías y hacia metodologías activas de aprendizaje. Son dos de los elementos que también estarán presentes en nuestra propuesta paradigmática, la tecno-holista, que justifica su existencia en base a la evolución constante del profesorado, que debe manejar con soltura competencias básicas actualizadas ligadas a modos de alfabetización audiovisual, tecnológica o digital sin pasar por alto la científica (Gómez, 2007: 46). Pero no olvidemos que en este modelo el 
alumnado adquiere una importancia en el sistema que podríamos interpretar de forma equilibrada a la del docente; uno de los papeles principales correspondería a los estudiantes que van a construir el conocimiento a partir de pautas o normas diseñados por el docente. El grado de responsabilidad frente al proceso de aprendizaje del alumno tiene especial protagonismo en estas metodologías activas, y también su grado de participación en experiencias y actividades que le permitan consensuar y discutir diferentes criterios sobre un mismo hecho. Si nos damos cuenta retornamos a la idea primitiva del agón griego con el que arrancábamos esta enumeración. Los paradigmas más actuales retoman lo ya experimentado anteriormente y vuelven a verse reflejados en planteamientos iniciales que sin embargo presumen de ser imprescindibles. Es una vuelta a la figura humana, un repensar la ciencia de forma cercana; el individuo siempre ha estado ahí, formando parte del proceso, más o menos ensombrecido por las metodologías educativas impuestas. Es hora de volver a darle el protagonismo que se merece, pero de forma ordenada, sin olvidar los procesos éticos de la educación y del sistema de enseñanza-aprendizaje, que en definitiva nos va convirtiendo en personas más instruidas, pero que a veces ha olvidado conducirnos a ser mejores personas.

1.2. El paradigma tecno-holista como una forma de completar un estilo educativo para la vida

Podríamos considerar a la educación holista como el nuevo paradigma educativo del presente siglo. La educación holística - del griego "holos" que significa totalidadnace a finales del siglo XX, en la década de los 90. "La educación holística -dice Carmen Cabestany, cofundadora de espacioholistico.es en una entrevista concedida a la Revista Esfinge- por su propia naturaleza, se integra y se nutre de diferentes tendencias pedagógicas y toma de cada una lo más valioso" "3 (2013), es decir vuelve la mirada a los clásicos pero reinterpreta el sistema educativo desde la creatividad, un elemento presente ahora pero sin precedentes que permite entender la educación desde perspectivas desconocidas o poco exploradas. Los holistas abordan la educación desde un planteamiento global, entendida como una "educación para la vida, que contempla al niño como un todo y no solo como un cerebro" (Gil, 2013) y atienden a criterios claros como los que se exponen a continuación. Por un lado la educación holista tiene como principal propósito el desarrollo humano y es que la visión humanista del proceso holista es uno de los sellos identificativos del nuevo sistema educativo, de ahí el "aprender a ser" como una de las premisas básicas de su pensamiento. Tal y como afirma Cabestany (2013), "el paradigma educativo holista se centra en el estudiante y están interesados en su formación y desarrollo como persona". Confían en la capacidad ilimitada del individuo para aprender de forma continua; no hay fronteras, normas u órdenes que limiten la capacidad de aprendizaje de un individuo y se llega a ese conocimiento a través de las vivencias personales, un proceso individual (ya que cada ser humano es único e irrepetible) que sin embargo se conduce a través de dinámicas activas, en muchas ocasiones grupales. La escuela con principios holísticos aplicados se convierte en un sistema vivo, parecido a una comunidad de aprendizaje, de ahí el "aprender a vivir juntos" como otra de las premi- 
sas en las que se sustenta el modelo, porque los holistas persiguen la formación y el desarrollo como persona, atendiendo a dos vertientes: por un lado, la relación que cada individuo tiene consigo mismo y por otro lado, considerándolo como ser en sociedad. Además el proceso educativo no tiene un mapa conceptual único ya que pueden darse por válidos multitud de caminos para llegar a alcanzar el conocimiento que se exige; lo importante es en definitiva "aprender a hacer"; la libertad que se espera del sistema, así como su flexibilidad, permiten que esta cualidad (que podría invitar al caos) se convierta en un punto favorable dentro del paradigma. En este tipo de sistemas holistas, el profesor y el estudiante se sitúan al mismo nivel, ya que ambos se encuentran en disposición de aprender (una de las novedades recae seguramente en el docente, que interviene en el proceso educativo de igual a igual), pero es que es importante que el estudiante interiorice y comprenda la filosofía del "aprender a aprender" como metodología de aprendizaje. Recordemos que para la educación holista, tal y como nos recuerdan Wompner y Fredy (2008), "aprender y enseñar no tienen la misma importancia, (...) el aprender tiene una importancia mucho mayor que el enseñar, el cual se realiza en el marco del aprender, por ello no cabe seguir hablando de un proceso de enseñanza-aprendizaje sino más bien de un aprendizaje holístico". No se olvidan los holistas de la educación ecológica y sistémica como una toma de conciencia planetaria y finalmente, y no menos importante, de la espiritualidad, a la que consideran el eje central de la experiencia de la totalidad así como del orden interno; se refieren, no obstante, a una espiritualidad que se aleja de forma tajante de creencias religiosas y dogmas y que se encarga de tener conciencia del ser dentro del mundo y del universo. Basándonos en los escritos de Ramón Gallegos Nava, uno de los pioneros de la educación holista en el mundo 4 podríamos destacar a la conciencia humana y su desarrollo como uno de los elementos fundamentales del paradigma. Esa conciencia, lejos de estar sola, se situaría entre el pensamiento y la inteligencia que son cualidades de la misma permitiendo por una parte el aprendizaje mecánico y por otra la posibilidad de observar la totalidad como unidad, ya que los holistas tienen en cuenta constantemente las interdependencias del todo integrado.

Pero la realidad presenta evoluciones que el modelo no parece destacar, o por lo menos asume de forma natural sin adecuar su importancia en el sistema. Nos referimos a la tecnología, presente y ligada a la evolución obligada de la educación a través de las TIC. Son precisamente las tecnologías de la información y de la comunicación el punto de apoyo principal de cualquier transformación propuesta en el mundo en el que vivimos. Es esta tecnología la que está obligando a la transformación forzada de competencias y saberes de los individuos, que en ocasiones no tienen tiempo de asimilar los conocimientos, dada la rapidez con la que cambian sus criterios de actuación. Estamos viviendo un momento histórico que permitirá mostrar en el futuro el giro brusco, acelerado y desconcertante que estamos sufriendo en la actualidad con el nacimiento de nuevas tecnologías o la transformación de las ya existentes. Lo que antes necesitaba de décadas para poder desarrollarse, ahora ve la luz escasamente en semanas o días, obligando al individuo a reciclarse de forma continua y a buscar fórmulas diferentes para integrarse en el sistema de aprendizaje, que ya defi- 
nió Carneiro (2012) como "el combustible de las nuevas economías", para alcanzar el conocimiento, que el mismo autor identifica como "el motor" de las mismas (15).

La tecnología parece que sólo se presupone presente en el holismo, sin embargo, la presencia del término se nos antoja imprescindible para entender un nuevo modelo de paradigma educativo encajado en un contexto histórico de actuación como el actual. Lo tecnológico se convierte en importante, imprescindible si queremos agilizar y afianzar los procesos de aprendizaje integrales, porque ayudan al desarrollo completo de la persona en sus diferentes facetas. La espiritualidad se presupone en el holismo porque aparece como un elemento innato a la naturaleza de su modelo, pero la tecnología queda tal vez difusa en el proceso de enseñanza-aprendizaje que se presupone como una excusa, solo como un paso más que invita al individuo a conocerse como un todo perfecto que puede lograr el objetivo del conocimiento por muchas y variadas vías. De ahí que nos atrevamos a puntualizar el término que interpreta el paradigma educativo holista, para pasar a denominarlo nuevo paradigma educativo tecno-holista. La variación terminológica, sin embargo, no afecta al diseño inicial del modelo que se mantiene intacto y que solo incorpora de forma evidente la tecnología que acompaña el desarrollo del mismo.

\section{Principales características educativas del espacio europeo de educación supe- rior}

El sistema educativo ha sufrido numerosas reformas con el paso de los años. La educación en cada país es un mundo y los métodos de enseñanza son diversos, incluso en un mismo país, como en España, encontramos algunas diferencias incluso en los curriculum escolares dependiendo de la Comunidad Autónoma en la que nos fijemos. No es de extrañar que surjan las comparaciones por ver qué país obtiene mejores resultados académicos. El camino educativo por configurar un Espacio Europeo de Educación Superior (EEES) ha sido y está siendo una tarea ardua, se ha tenido que romper con la educación tradicional basada en la mera transmisión de conocimientos para alcanzar lo que hoy en día denominamos paradigma tecno-holista donde la educación es un saber compartido mediado por las tecnologías y donde los valores y virtudes del hombre son fundamentales en el proceso educativo. Hasta la creación del Espacio Virtual Europeo de Enseñanza Superior, el sistema educativo se ha tenido que adaptar a numerosas declaraciones declaraciones - Carta Magna de las universidades europeas (1988), Declaración de La Soborna (1998), Declaración de Bolonia, (1999), I y II Convención Instituciones de Educación Superior (2001 y 2003), Conferencia de Ministros (2001, 2003 y 2009), etc- que han tenido como objetivo la mejora de los sistemas de enseñanza. 


\subsection{Las novedades introducidas por el espacio europeo de educación superior}

La palabra clave que cambia por completo la concepción educativa es calidad, una preocupación latente que surge dentro del ámbito educativo, social y político ante la inquietud de conseguir una Universidad de calidad acorde con las demandas de la sociedad del Siglo XXI que está supeditada al avance y al desarrollo tecnológico, donde el intercambio de información interfiere hasta en los modos de producción de una sociedad globalizada que debe dejar atrás el tradicional sistema de enseñanza que no cumple con las expectativas que de él se espera en esta nueva sociedad. Para poder lograr un cambio en la enseñanza superior ha sido necesario un movimiento unitario de los países miembros, es en la Unión Europea donde comienza a plantearse la creación de un espacio universitario común donde prime la máxima de la calidad y la competitividad.

En el año 1999 se constituye la Declaración de Bolonia de la que hoy forman parte activa 46 países, es sin duda, la base del proceso de convergencia en la Educación Superior y la que ha dado lugar a lo que hoy conocemos como Espacio Europeo de Educación Superior (EEES), donde las universidades deben adquirir una nueva concepción y una nueva forma de entender el trabajo:

Impulsar el aprendizaje activo y comprometido con el estudiante, a organizar la educación alrededor de las competencias que el estudiante debe alcanzar y, como fondo, a propiciar el desarrollo del pensamiento crítico que es, (...) el gran valor añadido y auténtico sello de identidad de la enseñanza universitaria (Calderón y Escalera, 2008: 238).

La enseñanza universitaria constituye el eje central de la economía y de la sociedad del conocimiento. Sin embargo, "el EEES no significa la homogeneización de las universidades en cuanto a sus misiones y fortalezas, sino que fija criterios comunes" (Arribas, Ochoa y Sánchez, 2009). De ahí que la Declaración de Bolonia esté compuesta por diversos principios que tratan de conseguir la convergencia en un entorno universitario europeo tomando como referencia las características de la sociedad vigente. Sistema de títulos de sencilla legibilidad y comparabilidad, sistema basado en dos ciclos principales de primer y segundo nivel, sistema de créditos ECTS, promoción de la movilidad para docentes y estudiantes, colaboración europea en la garantía de la calidad y promoción de las dimensiones europeas en lo que respecta al desarrollo curricular, colaboración interinstitucional, etc. (Declaración de Bolonia, 1999: 3) son algunos de los cambios introducidos por la declaración, que están siendo asumidos por las universidades españolas y que constituyen cambios en la reestructuración del hacer docente con la introducción de las Tecnologías de la Información y la Comunicación (TIC) y una modificación de los sistemas de planificación, evaluación, investigación, estructuras, herramientas informáticas, metodologías, infraestructuras, etc. Con estas iniciativas las universidades europeas ganan en "permeabilidad y transferencia de conocimientos, mediante la concreción de unas habilidades y competencias propias de cada titulación" (Pereyra, Luzón y Sevilla, 2006: 128). 
Si echamos la vista atrás, la universidad en España ha estado, hasta hace unos años, centrada en la profesionalización, olvidándose del papel tan importante que juega la investigación para el progreso. En el EEES ambos aspectos -profesionalización e investigación- no deben desligarse en el ámbito de la enseñanza, pues como afirman algunos autores "la Universidad además de la transmisión del conocimiento, también contribuye a su creación y aplicación social como base de nuevos conocimientos" (Pereyra, Luzón y Sevilla, 2006: 125). Así, los docentes universitarios, bajo la mirada de Bolonia, deben saber compaginar docencia e investigación.

El paso de una Sociedad del Conocimiento hacia una Sociedad del Aprendizaje supone para los docentes del EEES la adquisición de una serie de competencias hasta el momento no tenidas en cuenta durante los procesos de enseñanza/aprendizaje. Si atendemos a los escritos de Juana $\mathrm{M}^{\mathrm{a}}$ Madrid (2005), las nuevas competencias del profesorado serían cognitivas, meta-cognitivas, comunicativas, gerenciales de la enseñanza, sociales y afectivas (60), lo que daría lugar a cambios metodológicos donde el discente va a jugar un papel decisivo y activo en el proceso educativo. Para adaptarse a este nuevo marco del EEES el docente va a "dejar de ser tanto una fuente de información para pasar a tener un papel como facilitadores del conocimiento" (Rodríguez, 2009: 197), un aspecto que podría considerarse como la consecuencia de la llegada de las TIC al mundo de la docencia. Tan importantes son estas herramientas para el desempeño docente que a través de ellas se pueden construir espacios virtuales de enseñanza donde el proceso de comunicación se ve invertido y se pasa de una comunicación bidireccional a un espacio donde la adquisición de información y conocimiento es ilimitado y llega desde diversas fuentes (libro, ordenador, televisión, radio, etc.).

\subsection{La creación del espacio virtual europeo de enseñanza superior}

Las TIC propician el paso del aula física al aula virtual, con ellas el aprendizaje es más activo y el conocimiento sale de las cuatro paredes del aula para viajar y ser compartido en cualquier lugar del mundo con tan sólo un clic de ratón. Los espacios virtuales que vienen siendo tan empleados en los estudios de grado, posgrado, master y doctorado a través de las modalidades semipresenciales y online ofertadas por las universidades, no fueron tenidos en cuenta por la Declaración de Bolonia, por ello hasta el año 2000 no ha sido reconocido el papel tan importante que juegan las TIC en la enseñanza virtual.

$\mathrm{Al}$ amparo político ha surgido la iniciativa e-Europe para destacar el "el importante rol social y pedagógico" de las TIC, se pretende desde diversas instancias crear un Espacio Virtual Europeo de Enseñanza Superior (EVEES) donde se regule y aplique de forma eficaz el uso de las tecnologías (Gavari, 2006: 190). Algunas de las características de este espacio virtual pasa por la puesta en marcha del E-learning. Los principios de este tipo de espacios de enseñanza no son nuevos sino que valores como la movilidad, la igualdad de oportunidades, el aprendizaje a lo largo de toda la vida etc., ya formaban parte de las universidades a distancia. Sin embargo, esto principios se toman ahora como referencia para la creación de un espacio virtual de 
enseñanza donde los medios de comunicación y las tecnologías se convierten en un pilar básico de la universidades europeas a la hora de implantar metodologías activas en sus procesos de enseñanza/aprendizaje.

El nuevo alumnado que llega a las universidades es cada vez más tecnológico por lo que la Universidad debe adaptarse a una mayor demanda de la calidad, por ello cada vez es más frecuente oír hablar de la "Universidad Digital" (Arribas, Ochoa y Sánchez, 2009), aquella en la que la educación pasa de estar supeditada a la mera transmisión de conocimientos por parte del profesorado, a una educación donde el verdadero protagonista es el estudiante. Para ello, se fomentan los procesos cooperativos, comunicativos a través de las competencias generales de los títulos que cada vez son más importantes en el logro de una mayor empleabilidad y cualificación de los estudiantes de cara a su futura inclusión en el mundo laboral y social. Para conseguir esa máxima surgen nuevos paradigmas como el tecno-holista que trata de avivar el pensamiento crítico, la reflexión, la participación, que motive tanto a profesores como alumnos en el camino hacia una educación de calidad, sin olvidarse del uso de las TIC. Este paradigma se caracteriza por la unión de los aspectos relacionados con la personal social y el papel relevante que juegan las tecnologías en la vida cotidiana del ser humano. Ambos aspectos van a conformar el EVEES.

Las transformaciones y el uso de las tecnologías son cada vez más manifiestos al ser algo cada vez más cotidiano que supone la realización de cambios no sólo en las infraestructuras sino a nivel global en todos aquellos aspectos que confieren a la formación universitaria. Así, aparecen nuevas formas como el b-learning ${ }^{6}$, e-learning $^{7}$ y m-learning ${ }^{8}$ con las que aparecen nuevas metodologías que afectan a docentes y estudiantes debido a la inclusión del acceso a Internet, los campus virtuales, las videoconferencias, etc., en definitiva herramientas que transforman la concepción de la enseñanza tradicional. De ahí que aparezcan cambios significativos en el proceso educativo: mayor protagonismo del estudiante, desarrollo de competencias, nuevos conocimientos y habilidades, adquisición de herramientas, incorporación de las TIC, comunicación interpersonal y trabajo en equipo (Bosco y Rodríguez, 2008: 161-162). En este sentido, el EVEES va a aunar los objetivos que se marcan en el EEES con aquellos propios de la introducción de las nuevas tecnologías en los procesos educativos. No obstante, el sistema educativo se encuentra con serias dificultades a la hora de instaurar estos nuevos sistemas de enseñanza, ya que nos encontramos con evidentes diferencias educativas intergeneracionales, a lo que hay que sumarle un capital humano con déficit de formación, una población con un nivel educativo más bien bajo, lo que significa que la puesta en marcha e implantación del EVEES sea un proceso lento en nuestro país (Pereyra, Luzón y Sevilla, 2006: 129) y encontremos en pleno siglo XXI a docentes que se resisten al uso e implantación de las TIC en sus aulas. Esta afirmación queda reforzada con una de las conclusiones obtenidas en 2011 por el informe Competencia Mediática. Investigación sobre el grado de competencia de la ciudadanía en España donde se afirma que "tener estudios universitarios no garantiza la competencia mediática" (85). Así, una de las metas para poder implementar tanto el EEES como el EVEES debe ser el fomento de la competencia mediá- 
tica en los docentes y estudiantes, sino no se podrán desarrollar los nuevos procesos educativos que aquí venimos comentando.

\subsection{Las herramientas docentes en la ágora digital universitaria}

No podemos negar que la tecnología forma parte activa de nuestras vidas. Vivimos rodeados de pantallas, una realidad que traspasa las fronteras de la educación donde los usos que de los medios de comunicación y las TIC se puede hacer se presentan propios para el desarrollo de una educación más participativa, es lo que denomina el teórico Roberto Carvajal como Tecnología Educativa:

El conjunto de procedimientos, métodos herramientas y medios involucrados en un proceso sistemático con el objetivo de facilitar el desarrollo de las facultades individuales y de grupo, así como perfeccionar y afinar los sentidos humanos de todos los involucrados en el proceso de enseñanza aprendizaje (Lara, 2008: 139)

Ante esta definición es tarea sencilla extraer los beneficios que presentan este tipo de herramientas para el desarrollo de la actividad docente. No obstante, el uso de los mass media y las TIC requiere de un proceso de actualización constante por parte del profesorado, es decir, se hace necesario un aprendizaje continuo a lo largo de toda la vida, puesto que este tipo de tecnologías evolucionan constantemente. Sin embargo, no sólo es tarea del docente sino que las instituciones educativas deben realizar constantes cambios para actualizar sus sistemas e infraestructuras para poder cumplir con las demandas y expectativas de los denominados alumnos de la Generación Interactiva.

Los cambios que presenta la Ágora Digital del Siglo XXI ${ }^{9}$ atenderán no sólo a las infraestructuras sino que va a cambiar incluso la forma en que el docente difunde los conocimientos. Las pantallas se convierten ahora en elemento fundamental de las aulas y el libro tiene que aprender a convivir con el ordenador, la pizarra digital, el Ipad, las tabletas, los smartphone, los e-book, las consolas, etc. Así, los recursos multimedia de los que ahora dispone el docente para impartir una clase son cada vez más ilimitados:

Google Apps for Education: serie de herramientas vinculadas al correo electrónico que permiten diversas funciones desde estar en red, guardar, gestionar, editar y compartir documentos. Sus principales atractivos son Gmail, Drive, Docs, la Red Social y el sistema de Hangout.

Dropbox: este software ha revolucionado el mundo de la sincronización de archivos. Esta aplicación permite guardar, acceder y compartir carpetas y documentos con otras personas a través de cualquier ordenador con navegador web.

Skype: esta aplicación es idónea para la realización de vídeollamadas, llamadas de teléfono y envío de mensajes de forma instantánea con cualquier tipo de dispositivo. Su principal potencial para el ámbito educativo reside en su uso para la realización de videoconferencias grupales con hasta 10 personas a la vez de forma gratuita. 
Redes Sociales: la conectividad y el intercambio de información de las redes sociales ya ha llegado al ámbito educativo y redes como Facebook o Twitter se han convertido en plataformas idóneas para el trabajo en equipo, la movilización y el intercambio de ideas y conocimientos.

Mooc: con tan sólo 4 años de andadura los MOOC (Massive Open Online Courses) se están convirtiendo en una auténtica revolución en el ámbito universitario. El MOOC es un vídeo de corta duración que permite la transmisión de conocimientos a través de diversas plataformas que admiten su alojamiento. Estas pequeñas píldoras de conocimiento están dando lugar a cursos abiertos y masivos denominados xMOOC o a la aparición del cMOOC que introduce la variante de la conectividad.

Podcast de radio: sindicación de audio comprimido que permite crear y compartir grabaciones de audio en formato $\mathrm{mp} 3$ a través de diversas plataformas de Internet. $\mathrm{La}$ creación de un podcast es un proceso muy creativo y dinámico, en el que se deben tener en cuenta los principios y elementos que constituyen el denominado lenguaje radiofónico para la transmisión óptima de conocimientos.

Blog: es una plataforma web que permite la recopilación de textos y artículos que a su vez pueden ser enlazados con recursos multimedia (imágenes, vídeos y audios). En el ámbito educativo se viene utilizando una variante denominada Edublog cuya finalidad es educativa y va a permitir el apoyo a los procesos de enseñanza/aprendizaje e incluso la creación de comunidades educativas.

Cooperación, dinamismo o innovación son términos característicos del uso de estas herramientas en los nuevos procesos de enseñanza/aprendizaje de la educación superior. Sin embargo, para que el uso sea lo más adecuado posible institución y profesorado tienen que remar en una misma dirección y tener claros conceptos como interactividad, conectividad, servidor de aula, documentación, herramientas docentes y herramientas discentes (García, 2013) con el fin de hacer un uso lo más eficaz de las ventajas que presenta el uso de las TIC en el ámbito docente universitario.

\section{A modo de conclusión}

La sociedad tecnificada, demanda urgentemente una vuelta al origen educacional en el que el alumno y el docente se situaban juntos frente al conocimiento, y en el que la meta no era saber más sino ser mejor persona. El paradigma tecno-holista aparece como un modelo ideal que funde los elementos fundamentales de la tecnología con el holismo.

Para que en este nuevo modelo educativo se produzca es necesario que el docente tenga adquiridos un amplio repertorio basado en lo cultural, lo científico y lo profesional; pero además, estos conocimientos deberían estar acompañados de una nueva concepción del proceso metodológico de enseñanza/aprendizaje en el que las TIC son protagonistas. Para ello, también va a necesitar atender a aspectos metodológi- 
cos, organizativos y formativos, con el fin de desarrollar una docencia globalizadora donde la comunicación entre docente/discente deja de ser bidireccional.

La transmisión del conocimiento en el ámbito de la docencia universitaria está cambiando, debido entre otros aspectos a las transformaciones de infraestructuras que exige el nuevo Espacio Europeo de Educación Superior, que muestra una nueva configuración del ágora tradicional hacia lo digital.

\section{Bibliografía}

4.1 Libros:

COLOM, A. J. (2002): La (de)construcción del conocimiento pedagógico. Paidós. Barcelona.

FERRÉS I PRATS, J., GARCÍA MATILLA, A., AGUADED GÓMEZ, J. I., FERNÁNDEZ CAVIA, J., FIGUERAS, M. y BLANES, M. (2011): Competencia Mediática. Investigación sobre el grado de competencia de la ciudadanía en España. Ministerio de Educación e Instituto de Tecnologías Educativas. Madrid. MONTAGU, A. (1958): The cultured man. World. Cleveland.

4.2 Capítulos o artículos en libros o revistas en papel:

LLORENTE CEJUDO, M.C. y MARÍN DÍAZ, V. (2010). “Tecnologías móviles para la enseñanza". En SOLANO FERNÁNDEZ, I. M. (Coord.). (2010). Podcast educativo. Aplicaciones y orientaciones del m-learning para la enseñanza. Sevi1la: Eduforma. p.13-36

4.3 Artículos en publicaciones web:

ARRIBAS ALONSO, Y., OCHOA MENDOZA, I. y SÁNCHEZ GODOY, T. (2009): "Hacia la Universidad Digital 2010. Las TIC como palanca de cambio". Telos, $\mathrm{n}^{\circ}$ 78. Disponible en http://goo.gl/0sbHbB [17-09-2013]

AYUSTE GONZÁlEZ, A. y TRILLA BERNET, J. (2005): "Pedagogías de la modernidad y discursos postmodernos sobre la educación". En: Revista de Educación, $\mathrm{n}^{\mathrm{o}}$. 336, Madrid. p. 219-248. Disponible en http://goo.gl/KkpoHc [03-092013]

BENITO, M. (2009): "Desafíos pedagógicos de la escuela virtual. Las TIC y los nuevos paradigmas educativos". Revista Telos, $\mathrm{n}^{\circ}$. 78. Disponible en http://goo. $\mathrm{gl} / \mathrm{dZPTJK}$ [11-09-2013]

BOSCO PANIAGUA, M. A. y RODRÍGUEZ GÓMEZ, D. (2008): “Docencia virtual y aprendizaje autónomo: algunas contribuciones al Espacio Europeo de Educación Superior". En: RIED. Vol. 11, no . 1. p. 157-182. Disponible en http://goo.gl/ n3ssWh [03-09-2013] 
CALDERÓN PATIER, C. y ESCALERA IZQUIERDO, G. (2008): "La evaluación de la docencia ante el reto del Espacio Europeo de Educación Superior (EEES)". En: Educación XXI. p. 237-256. Disponible en http://goo.gl/xmLGoj [17-09-2013]

CARNEIRO, R. (2012): "Las TIC y los nuevos paradigmas educativos: la transformación de la escuela en una sociedad que se transforma". Secretaría de Educación del Gobierno de México. Disponible en http://goo.gl/hSkp2N [11-09-2013]

DECLARACIÓN DE BOLONIA. (1999): Disponible en http://goo.gl/bNVcYA [11-09-2013]

FUGONAZZI, T. (2011): "Viejo paradigma vs Nuevo paradigma". En: Revista Namaste. Disponible en http://goo.gl/9RUa7R [03-09-2013]

GARCÍA, A. (2013): "Hablamos de Educación: el Aula Digital". Blog Tiching. Disponible en http://goo.gl/tHa2x [17-09-2013]

GAVARI, E. (2006): "Los principios rectores del Espacio Europeo de Educación Superior". En: Revista Electrónica Teoría de la Educación. Educación y Cultura en la Sociedad de la Información, $\mathrm{n}^{\circ}$ 2. p. 185-197. Disponible en http://goo. gl/0GA7Zs [03-09-2013]

GIL, H. (2013): "Educación holística: una educación para los nuevos tiempos". Esfinge. Apuntes para un pensamiento diferente. Disponible en http://goo.gl/ tJAkdC [17-09-2013]

GÓMEZ HERNÁNDEZ, J. A. (2007): “Alfabetización informacional: cuestiones básicas". Anuario ThinkEPI. p. 43-50. Disponible en http://goo.g1/3fkrOS [03-092013]

KAHALE CARRILLO, D. T. (2011): "Virtual Rooms. Instrumentos de enseñanza en la educación a distancia universitaria". En: Icono 14, vol. 9, n 2. p. 138-150. Disponible en http://goo.gl/DqQQdk [03-09-2013]

LARA TREVIÑO, J. I. (2008): "Percepción de profesores de universidad pública sobre el uso de las tecnologías de información aplicadas a la educación". En: Entelequia. Revista Interdisciplinar, $\mathrm{n}^{\circ}$ 6. p. 123-175. Disponible en http://goo. gl/5efHtP [17-09-2013]

MADRID IZQUIERDO, J. Ma . (2005): "La formación y la evaluación docente del profesorado universitario ante el espacio europeo de educación superior”. En: Educatio, ${ }^{0}$ 23. p. 49-68. Disponible en http://goo.gl/qJuZrS [17-09-2013]

MARTÍNEZ MIGUELEZ, M. (2009): "Un nuevo paradigma para la Educación". BuenasTareas.com. Disponible en http://goo.gl/ZQ0MHz [03-09-2013]

MONTOYA GUERREROS, J. (2010): "Ramón Gallegos y el Paradigma de la Educación Holista". BuenasTareas.com. Disponible en http://goo.gl/EmyNgn [17-092013]

PEREYRA, Miguel A., LUZÓN TRUJILlO, A. y SEVILLA MERINO, D. (2006): "Las universidades españolas y el proceso de construcción del Espacio Europeo de Educación Superior. Limitaciones y perspectivas de cambio". En: Revista Española de Educación Comparada, $\mathrm{n}^{\mathrm{o}}$ 12. p. 113-143. Disponible en http://goo. gl/40Oy9c [11-09-2013]

RODRÍGUEZ IZQUIERDO, R. M. (2009): “Innovación metodológica docente en el marco del espacio de europeo de educación superior: Algunas reflexiones desde 
los retos de la sociedad del conocimiento". En: XXI. Revista de Educación, $\mathrm{n}^{\circ} 11$. p. 195-206. Disponible en http://goo.gl/Zelr6L [03-09-2013]

ULlOQUE, G. (2001): "Tecnología, ciencia y el pez de Mcluhan. Concepciones sobre la tecnología y su relación con la ciencia". En: Revista Iberoamericana de Educación, $\mathrm{n}^{\mathrm{o}}$ 54. Disponible en http://goo.gl/XAO3Oz [11-09-2013]

WOMPBER, G. y FREDY, H. (2008): "Educación Integral: la llave para una nueva era". En: Entelequia. Revista Interdisciplinar, no. 6. p. 177-192. Disponible en http://goo.g1/P1Qdj2 [11-09-2013]

\section{Notas}

1 Serían pocos los teóricos, filósofos, pedagogos o educadores que se posicionarían de forma clara en uno u otro modelo de forma exclusiva. La tendencia, no olvidemos, es reconocer la versatilidad del pensamiento que se amolda con facilidad a criterios de unos u otros modelos.

2 Aunque, tal y como recoge el profesor Ulloque (2009), no se encuentran referencias bibliográficas que den constancia de ello, tal vez por referirse a una reflexión oral que el estudioso lanzaba en clase a sus alumnos.

3 En esa entrevista citada, Cabestany asegura que son muchas las tradiciones filosóficas o pedagógicas que respaldan al holismo: "desde Sócrates y Platón, pasando por J. Rousseau, Husserl, Teilhard de Chardin, Pestalozzi, Piaget, la Filosofía perenne, la Pedagogía ancestral, Paulo Freire, María Montessori, la Pedagogía Waldorf, la Pedagogía Sistémica..."(2013)

4 Se recomienda la lectura del ensayo de Jesús Montoya Guerrero "Ramón Gallegos y el Paradigma de la Educación Holista" (2010) en el que hace una pequeña reflexión sobre algunos de los libros que componen la producción de Ramón Gallegos Nava, que definen tanto los fundamentos históricos de su obra como la propuesta de su modelo educativo en la educación holista.

5 Las principales acciones de este programa europeo fueron la Alfabetización Digital, los campus virtuales, el hermanamiento electrónico entre centros, la formación del profesorado y la promoción del propio programa en Europa (Gavari, 2006: 190).

6 Esta terminología hace referencia a aquella enseñanza combinada "en la que se entrecruzan tanto elementos presenciales como virtuales" (Kahale, 2011: 140), es por tanto la unión de la clases tradicionales con las virtuales. En definitiva, lo que en algunas universidades se viene denominando Semipresencialidad.

7 Normalmente cuando se hace referencia al e-learning, los autores atienden a aquella enseñanza no presencial que se suele considerar on-line, ya que necesita de medios y plataformas tecnológicas para su desarrollo a través de la red de Internet.

8 Más conocido como Mobile Learning, este sistema de enseñanza es la unión de todos los anteriormente citados y se caracteriza por el desarrollo de un aprendizaje electrónico móvil. Como señalan las autoras $\mathrm{M}^{\mathrm{a}}$ del Carmen Llorente y Verónica Marín (2010) el M-learning está compuesto por términos como: movilidad, espontaneidad, objetos, intimidad situada, conectividad, informal, General Packet Radio Service, G3, bluetooth, redes, aprendizaje situado, situaciones reales, constructivismo, colaboración, etc., estando este sistema "ubicado en ambientes y tiempos más independientes" (p. 15).

9 Este espacio, que bien podrá ser real o virtual, estará compuesto de un equipo adecuado atendiendo a aspectos disciplinares, didácticos y tecnológicos donde poder llevar a cabo una acción educativa 
debidamente planificada. El profesor Albert García (2013) considera fundamentales los componentes metodológicos, de entorno web, de explicación, de gestión de aula, de datos y de entorno grupal de aprendizaje para poder desarrollar una acción educativa óptima en las enseñanzas que incluyen a las TIC.

\section{Las autoras}

Irene Melgarejo Moreno. Doctoranda en Ciencias de la Comunicación en la Universidad Católica San Antonio de Murcia, Diplomada en Estudios Avanzados (DEA) y Licenciada en Comunicación Audiovisual con especialización en radio y televisión (UCAM). Profesora de las asignaturas "Sociedad, Familia y Escuela" y "Medios, materiales y Nuevas Tecnologías aplicadas a la Educación" en los Grados de Educación Infantil y Primaria de la UCAM. Miembro del Grupo de Investigación Comunicación y Menores y socia de la Asociación Internacional para la Investigación sobre Infancia, Juventud y Comunicación (KIDS\&COM), sigue las líneas de investigación relacionadas con los medios de comunicación y la infancia. Ha participado en reuniones científicas y congresos internacionales, es coautora de varios libros y ha realizado diversas publicaciones en revistas científicas de comunicación.

María del Mar Rodríguez Rosell es Doctora en CC. de la Información por la Universidad Complutense de Madrid, acreditada por la Aneca como Profesor Contratado Doctor y Licenciada en Comunicación Audiovisual (UCM). Profesora Adjunta de diferentes asignaturas en los Grados de Comunicación y Educación Infantil de la UCAM donde ejerce de Secretaria Académica de la Facultad de CC. Sociales y de la Comunicación y donde es Subdirectora de la revista científica Sphera Publica; desde 2005 es Directora del Grupo de Investigación Comunicación y Menores. Sus líneas de investigación se dirigen a profundizar sobre la relación de los Medios de Comunicación y los menores materializadas en diversas publicaciones en revistas científicas de comunicación. Directora del Blog "Comunicación y Menores" y Socia de la Asociación Internacional para la Investigación sobre Infancia, Juventud y Comunicación (KIDS\&COM). 